\title{
EDITORIALE
}

\section{Performance e Execution oppure Performance è Execution?}

\author{
Maria Pia Maraghini* , Angelo Riccaboni*
}

\begin{abstract}
Performance and Execution or Performance is Execution?

The term 'performance' is progressively recovering its lost dignity (at least in recent years).

In particular, it correlates perfectly - much better than its 'substitutes' - to the concept of execution, today more than ever at the center of the debate around the theme of how companies should be managed and governed. This highlights the pivotal role of the 'performance' within the overall Management (or Performance Management) System.

Recognizing that, this issue completely focuses on the theme of business 'performance', with the aim of highlighting its role of an effective (as well as timely) planning, programming, measurement, analysis and evaluation of company performance in supporting corporate governance.
\end{abstract}

Keywords: performance, measurement, shared value, sustainable development, execution, performance management.

Dipartimento di Studi Aziendali e Giuridici, Università degli Studi di Siena, e-mail: mariapia.maraghini@unisi.it; angelo.riccaboni@unisi.it.

Management Control (ISSN 2239-0391, ISSNe 2239-4397), 2019, 2

DOI: 10.3280/MACO2019-002001 


\section{Introduzione}

Il termine "performance" sta diventando sempre più desueto in ambito aziendale. Anche andando ad analizzare l'impiego del vocabolo nell'ambito della letteratura accademica nazionale, si rileva un progressivo minore ricorso allo stesso.

In particolare, inserendo le parole chiave "performance" e "misurazione delle performance" nel motore di ricerca Google Scholar (liberamente accessibile) vengono evidenziate citazioni dei due termini all'interno del numero di prodotti rappresentato in Figura 1 (distinto per ogni anno dell'ultimo quinquennio).

Figura 1 - Il ricorso ai termini "performance" e "misurazione delle performance" nella letteratura accademica nazionale (quinquennio 2015-2019)

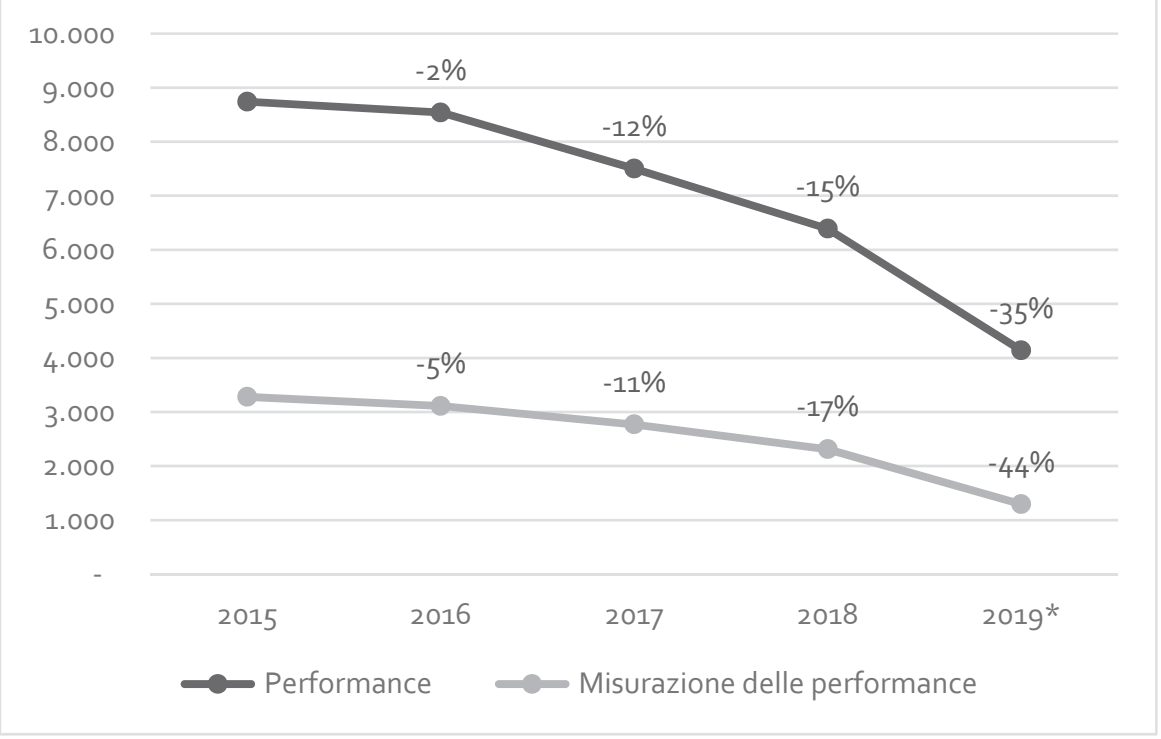

Rilevazione: Google Scholar (giugno 2019)

* Avendo l'analisi il mero fine di evidenziare la tendenza dell'andamento dell'impiego dei due termini, il valore 2019 (relativo solo ai primi sei mesi dell'anno) è stato semplicemente moltiplicato per 2 . 
Considerata la specifica finalità conoscitiva, nella ricerca sono state selezionate solo le pagine in italiano ed esclusi brevetti e citazioni. Tuttavia, anche analizzando il dato complessivo (ovvero non limitando la ricerca alle sole pagine in italiano), si evidenzia comunque un medesimo andamento decrescente (addirittura più marcato nei primi quattro anni del periodo preso in considerazione) sia considerando, che escludendo, brevetti e citazioni (rispettivamente, si rilevano i seguenti andamenti delle citazioni del termine "performance": 2016: -18\% e -22\%; 2017: -36\% e -35\% ; 2018: -57\% e 56\%; 2019*: -3\% e -13\% e di quello di "performace measurement": 2016: 20\% e - $21 \%$; 2017: -43\% e -41\% ; 2018: -60\% e -58\%; 2019*: -22\% e -17\%).

Pur nella consapevolezza che Google Scholar comprende tutti i settori della ricerca scientifica, la tendenza evidenziata può ricondursi anche al solo ambito economico-aziendale, il quale comprende comunque circa i $3 / 4$ delle citazioni inerenti la "performance" (si veda, ad esempio, la Figura 2).

Figura 2 - Distinzione delle citazioni inerenti la "performance" nella letteratura accademica nazionale (anno 2018)

\begin{tabular}{|l|c|}
\hline Economica & $26 \%$ \\
\hline Organizzativa & $14 \%$ \\
\hline Aziendale & $13 \%$ \\
\hline Finanziaria & $11 \%$ \\
\hline Sanitaria & $12 \%$ \\
\hline Individuale & $15 \%$ \\
\hline Sportiva & $7 \%$ \\
\hline Altro & $1 \%$ \\
\hline
\end{tabular}

Una simile "disaffezione" al termine "performance" può essere ricollegata ad un duplice ordine di motivi.

1. In primo luogo, essa può ricondursi alla volontà di "distaccarsi" dall'accezione comune del termine, ovvero dai tradizionali modelli di misurazione dei risultati aziendali (Riccaboni, 1999), aventi ad oggetto le performance "contabili" (economico-finanziarie e patrimoniali). A partire dalla fine del secolo scorso, infatti, i fini stessi dell'impresa sono stati oggetto di ridefinizione, cui è inevitabilmente seguito un momento di profondo ripensamento anche del tema della misurazione delle performance organizzative (Pozza, 2000). Ai risultati economico-finanziari e patrimoniali quali fine dell'agire aziendale, si è cominciato dunque a preferire il concetto di "valore" (Catturi, 2003) o, meglio, di "shared value" (Porter, 
Kramer, 2006 e 2011) e quello di "sviluppo sostenibile" (Pezzoli, 1997; Busco, Riccaboni, 2010; Balkyte, Tvaronavičienè, 2010; Busco, Frigo, Riccaboni, 2017; Maraghini, 2019).

2. Inoltre, il termine "performance" viene tradizionalmente correlato ad una sfumatura valutativa positiva (Accademia della Crusca, 2014). Si comprende dunque come gli andamenti economico-finanziari dell'ultimo decennio abbiano scoraggiato il ricorso al termine, al quale sono stati preferiti vocaboli maggiormente «neutri» quali "andamenti" o "risultati" (che possono essere sia positivi che negativi).

In realtà, ci sembra proprio questo il momento di un ritorno in auge del termine "performance", il quale merita di recuperare la dignità perduta.

a. Innanzitutto, andando a recuperare l'origine etimologica del termine, il quale "sarebbe giunto in italiano dall'inglese, e prima ancora all'inglese dal medio francese parformance (Zingarelli, 2013), a sua volta deverbale dall'antico francese parformer". Al "performance" primonovecentesco sarebbe stato possibile sostituire in modo quasi indolore il termine "prestazione" o quello di "rendimento" (Accademia della Crusca, 2014), siano dunque essi sia positivi che negativi, materiali o "intangibili", o di qualsivoglia natura (economico-finanziaria, ambientale, sociale). Vengono quindi "azzerati" entrambi i motivi alla base della progressiva reticenza sin qui evidenziata nei confronti dell'impiego del termine "performance".

b. Non solo, "continuando a percorrere a ritroso la storia del termine si finisce per riconoscerne un lontano antenato nel verbo tardo latino performāre", che significa 'dare forma definitiva', modellare. Inoltre, sembra che lo sviluppo semantico del termine sia stato incoraggiato e arricchito dall'antica interferenza tra il latino (e romanzo) formare e la radice germanica frumjan (che in un'accezione ormai defunta - ma storicamente molto importante - significava 'completare, soddisfare, portare a termine'), e anche dell'italiano fornire, nel suo senso di 'portare a compimento, mettere in esecuzione, terminare' ${ }^{1}$.

La performance rappresenta dunque il momento della «realizzazione», del «compimento» (o «concretamento») del fine aziendale, ovvero dei suoi obiettivi strategici.

${ }^{1}$ Il termine "performance" assume dunque dei caratteri "esotici", in quanto è privo di una cittadinanza precisa, il cui significato è stato tuttavia capace di determinarsi in virtù delle interferenze e dei passaggi a nuovi ambiti d'uso o a lingue differenti (Accademia della Crusca, 2014). 
In tal senso, l'espressione «performance» si correla perfettamente - molto meglio dei suoi "sostituti" (ovvero i già richiamati termini di "prestazione", "rendimento" o quello di "andamento") - al concetto di execution, oggi più che mai al centro del dibattito attorno al tema del come le imprese debbano essere gestite e governate (Bossidy, Charan, 2002; Kaplan, Norton, 2008; Economist, 2017). L'execution rappresenta infatti l'effettiva implementazione della strategia aziendale, ovvero il 'dare forma' allo 'shared value', $\mathrm{o}$, in altri termini, il 'portare a compimento' gli obiettivi di sviluppo sostenibile.

Si evidenzia così il ruolo cardine della «performance» nell'ambito del complessivo sistema di gestione aziendale - cosiddetto Management system o Ciclo di gestione delle performance (Kaplan, Norton, 2008; v. Figura 3).

Figura 3 - Il Management System

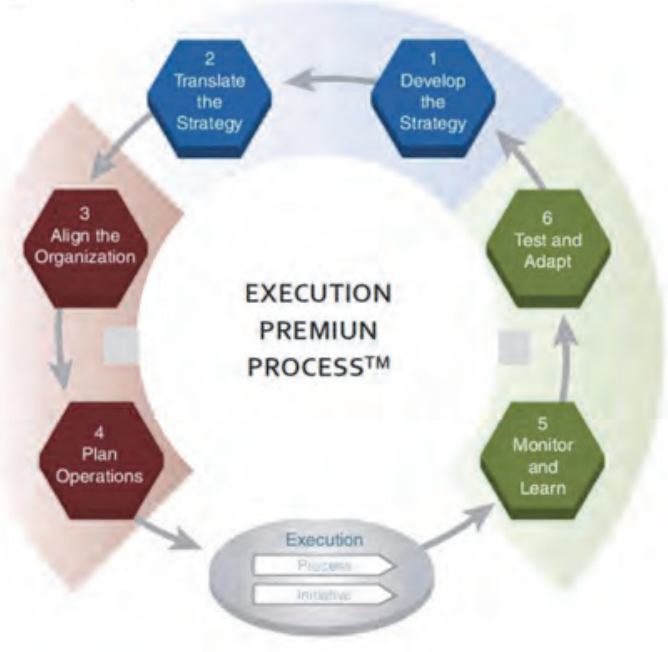

Fonte: Kaplan, Norton, 2009.

In effetti, perfino una strategia organizzativa ben formulata assume ben poco valore qualora non altrettanto efficacemente implementata. Ne deriva che la stessa valutazione della qualità di una strategia dipende dal come e quando della sua adozione, ovvero dalle performance che da essa si originano e da cui, al tempo stesso, è determinata.

La «performance», dunque, torna ad essere il principale focus della gestione aziendale - ovvero lo 'snodo' fondamentale del suo ciclo. Conseguentemente, essa dovrebbe anche divenire un tema sempre più centrale, sia nei contesti operativi che nella letteratura economica in materia. 


\section{Il contenuto del fascicolo}

Riconoscendo tutto ciò, il presente fascicolo si incentrata completamente sul tema delle «performance» in ambito aziendale, con l'obiettivo di evidenziare il ruolo della loro efficace (nonché tempestiva e bilanciata) pianificazione, programmazione, misurazione, analisi e valutazione a supporto del governo delle imprese di qualunque natura, dimensione e/o ambito di attività.

In particolare, considerate le ovvie necessità di restrizione del campo di indagine, $i$ contributi accolti in questo numero della Rivista si concentrano attorno a due principali obiettivi:

I. far emergere le esigenze di integrazione/allineamento con tutti gli altri sistemi e le dimensioni di analisi dell'agire organizzativo, affinché possano essere perseguiti livelli di performance più elevati;

II. focalizzare l'attenzione nell'ambito dei processi di riforma delle amministrazioni pubbliche.

Ancor più nello specifico, i primi tre articoli si concentrano nell'approfondire le interazioni fra la "performance» aziendale ed i sistemi di incentivazione (contributo curato da Davide Rizzotti), nonché con i sistemi di gestione strategica (con maggiore precisione, balanced scorecard), i quali vengono analizzati, in particolare, incentrandosi sul governo del grado di incertezza ambientale percepita (lavoro a cura di Antonio Costantini, Filippo Zanin e Marco Fasan) o delle strategie ambientali (contributo di Rosita Capurro).

Nel rispetto dell'intento generale della Rivista di combinare differenti tipologie di ricerca, mentre il primo lavoro si avvale di un esperimento di laboratorio (condotto con un consistente numero di studenti universitari magistrali) per testare le ipotesi di ricerca avanzate, l'analisi empirica del secondo si basa su di un questionario somministrato ad un campione rappresentativo di imprese italiane di grandi dimensioni attive nel settore manifatturiero. Il terzo articolo contiene invece la proposta di uno strumento utile per la gestione ed il monitoraggio delle strategie ambientali.

L'altra sezione del fascicolo focalizza invece l'attenzione in riferimento ad una specifica tipologia di aziende, ovvero alle amministrazioni pubbliche italiane e, più nello specifico, nell'ambito delle Università, delle scuole pubbliche e dei Comuni.

Sempre nell'intento di combinare differenti tipologie di ricerca, mentre il contributo di Alberto Ezza, Nicoletta Fadda, Gianfranco Pischedda e Ludovico Marinò si basa su di un'analisi longitudinale degli open data elaborati dall'Ufficio Statistico del MIUR, il lavoro di Domenico Raucci, Stefano 
Agostinone e Erika Di Santo si avvale anche di un'indagine esplorativo-descrittiva riferita agli anni 2011-2016 su di un campione di 2.000 scuole pubbliche italiane. Infine, l'articolo di Maria Teresa Nardo, Antonio Nisio e Patrizia Romanazzi impiega invece il metodo del multiple-case study research confrontando le performance di tre Comuni di grandi dimensioni, considerati best practices in materia di programmazione economico-finanziaria.

In definitiva, dando evidenza al momento di "realizzazione" degli obiettivi strategici aziendali, i contributi raccolti nel presente fascicolo offrono preziosi suggerimenti in merito alle modalità di gestione da poter implementare (in differenti contesti) affinché possano essere perseguiti più elevati livelli di «performance».

\section{Ringraziamenti}

Si ringrazia la Dott.ssa Alessandra Goti per la preziosa assistenza editoriale fornita nella preparazione di questo numero.

\section{Bibliografia}

Accademia della Crusca (2014), Intorno alla performance, -- www.accademiadellacrusca.it/it/lingua-italiana/consulenza-linguistica/domande-risposte/intorno-performance.

Balkytė A., Tvaronavičienė M. (2010), Perception of competitiveness in the context of sustainable development: facets of «sustainable competitiveness», Journal of Business Economics and Management, 11, 2, pp. 341-365.

Bossidy L., Charan R. (2002), Execution: The Discipline of Getting Things Done.

Busco C., Frigo M., Riccaboni A. (2017), Sustainability Development Goals: Integrating Sustainability Initiatives With Long-Term Value Creation, in Strategic Finance.

Busco C., Riccaboni A. (2010), Governo e gestione delle performance d'impresa, Milano, Pearson.

Catturi G. (2003), L'azienda universal, Padova, CEDAM.

Economist (2017), Perché le strategie non hanno successo? Colpa dell'execution.

Kaplan R., Norton D. (2008), The execution premium: linking strategy to operations for competitive advantage.

Maraghini M.P. (2019), Sostenibilità e governo aziendale. Parola d'ordine: integrazione!, in Bastianoni S. (a cura di), Manuale del corso di Sostenibilità, Milano (in press), Milano, FrancoAngeli.

Pezzoli K. (1997), Sustainable Development: A Transdisciplinary Overview of the Literature, Journal of Environmental Planning and Management, 40, 5, pp. 549-574.

Porter M.E., Kramer M.R. (2006), Strategy \& Society: The Link between Competitive Advantage and Corporate Social Responsibility, Harvard Business Review, 84, 12, pp. 7892.

Porter M.E., Kramer M.R. (2011), Creating Shared Value, Harvard Business Review, 89, 1/2, pp. 62-77.

Pozza L. (2000), La misurazione della performance d'impresa. Strumenti e schemi, Milano, EGEA. 
Maria Pia Maraghini, Angelo Riccaboni

Riccaboni A. (1999), Performance ed incentivi, Padova, Cedam.

Kaplan R.S., Norton D.P. (2008), Governare il Management System, Harvard Business Review, Gennaio-Febbraio, pp. 45-63.

Kaplan R.S., Norton D.P. (2009), Execution Premium. Applicare la strategia per il vantaggio competitivo, Milano, Etas RCS.

Zingarelli (2013), Dizionario Etimologico della Lingua Italiana. mezzo effettuata e la sua messa a disposizione di terzi, sia in forma gratuita sia a pagamento. 